\title{
Mapping Static and Dynamic Crack-Tip Deformations Using Reflection-Mode Digital Gradient Sensing: Applications to Mode-I and Mixed-Mode Fracture
}

\author{
Amith S. Jain ${ }^{1} \cdot$ Hareesh V. Tippur ${ }^{1}$
}

Received: 3 March 2015 / Accepted: 30 May 2015 / Published online: 9 June 2015

(C) Society for Experimental Mechanics, Inc 2015

\begin{abstract}
The reflection-mode digital gradient sensing ( $\mathrm{r}-$ DGS) method is extended for visualizing and quantifying crack-tip deformations in solids under quasi-static and dynamic loading conditions. The r-DGS technique employs digital image correlation principles to quantify two orthogonal surface slopes simultaneously in specularly reflective solids by measuring small deflections of light rays. Here, for the first time, r-DGS is implemented to study both mode-I and mixed-mode (I/II) problems and evaluate fracture parameters. Under dynamic loading conditions, r-DGS is employed in conjunction with highspeed digital photography to map surface slopes in edge cracked plates subjected to one-point impact. The measured surface slopes are used to successfully evaluate instantaneous stress intensity factor histories by combining measurements with the corresponding asymptotic crack-tip fields and performing over-deterministic least-squares analyses. Finite element computations are also used to complement experimental measurements as needed.
\end{abstract}

Keywords Optical metrology - Surface slopes · Digital image correlation $\cdot$ Stress wave propagation $\cdot$ High-speed digital photography $\cdot$ Fracture mechanics

Hareesh V. Tippur

tippuhv@auburn.edu

1 Department of Mechanical Engineering, Auburn University, Auburn, AL 36849, USA

\section{Introduction}

Measurement of stresses, strains, and deformations is at the heart of experimental mechanics studies. Over the years, this community of researchers has introduced many different measurement techniques, both point-wise and fullfield, to understand the underlying mechanics of materials. Point-wise techniques such as strain gages and fiber optic sensors have been popular due to their cost-effectiveness and simpler signal processing requirements. The full-field techniques on the other hand generally involve spatiallyrich 2-D and 3-D optical signals and have contributed to several fundamental breakthroughs in mechanics in general and stress concentration studies related to material fracture/failure in particular [1, 2]. The full-field nature of measurement techniques generally involves elaborate data analyses with varying degrees of user intervention. The optical techniques can be further classified into incoherent and coherent types. Photoelasticity [3-5], moiré methods [6-8], optical caustics $[9,10]$ generally use incoherent light from extended sources and require special surface and/or material characteristics such as birefringence, reflectivity, printed gratings, etc., for implementation. Others such as speckle photography [11], holographic interferometry [12], Twyman-Green interferometry [13], speckle shearing interferometry [14], coherent gradient sensing [15-17] all use coherent laser-light.

In the 1980s digital image correlation $[18,19]$ and white light speckle photography $[20,21]$ methods were introduced as full-field experimental mechanics tools. They employ random speckles, typically created by misting the surface with black and white paint in the absence of a natural texture, to monitor motion of material points. The latter used Fourier domain correlation based on optical spatial filtering principles whereas the former employed 
spatial domain digital correlation. The digital image correlation or DIC methods have rapidly evolved in the past decade or so from being a 2-D surface deformation measurement approach to sophisticated 3-D measurement technique employing stereo-imaging principles. Other advances in digital image processing methods, spectral augmentation and resolution enhancement of optical sensors along with an exponential growth in computer processing power have enabled volumetric measurements as well. The relative ease of implementation and possibilities for automation has made DIC methods popular in industrial and research settings alike.

In this context, a technique called digital gradient sensing (DGS) method based on 2-D digital image correlation, has been recently introduced for studying phase (transparent) objects [22, 23] and reflective solids [24] by measuring small angular deflections of light rays caused by inhomogeneities, defects or deformation of solids. In the former, the method has been successfully demonstrated by quantifying two orthogonal stress gradients near stress concentrations in solids whereas the latter has been demonstrated by measuring full-field orthogonal surface slopes of thin plates subjected to flexure. The purpose of the current work is to extend the reflection-mode DGS (or, simply r-DGS) method to quasi-static and dynamic fracture mechanics studies. Visualization of time-resolved deformations near mode-I and mixed-mode (mode I/II) cracks for quantifying fracture parameters are among the goals of this work.

\section{Optical Setup and Working Principle}

The schematic of the optical setup used for reflection-mode DGS (or, r-DGS) is shown in Fig. 1. It consists of a specularly reflective planar object (specimen), a (50/50) beam splitter, a randomly speckled/textured planar target and a digital recording device (camera). The speckle pattern on the target is prepared by decorating a planar surface with alternative black/white mists of paint. The nature of speckles used for a given application depends on the camera parameters (pixel and gray scale resolutions, camera gain, numerical aperture of the lens), image magnification and measurement resolution needed.

The optical arrangement is such that the digital camera is used to record speckles on the target plane via the reflective specimen and the beam splitter. In the undeformed or the reference state of the specimen, a generic point $\mathrm{P}$ on the target is photographed through a point $\mathrm{O}$ on the specimen surface. When the specimen deforms, the point $\mathrm{O}$ moves to $\mathrm{O}^{\prime}$ with an out-of-plane displacement $w$ in the $z$-direction (the optical axis). This results in the
Fig. 1 Schematic depicting the experimental setup and working principle of reflection-mode DGS (r-DGS) method

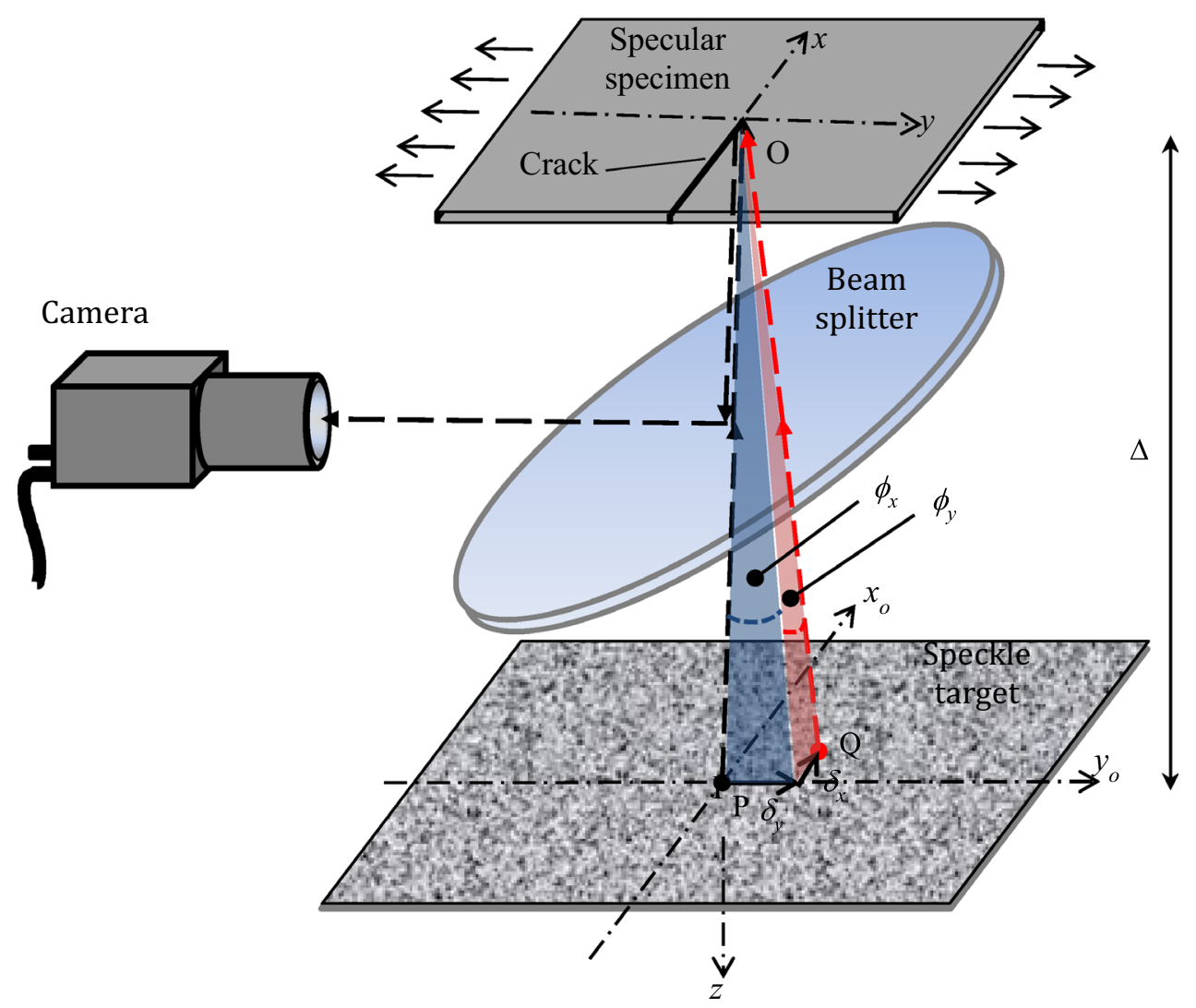


camera photographing a neighboring point $\mathrm{Q}$ on the target in the deformed state of the specimen. By correlating these two images corresponding to the reference and deformed states of the specimen, the local displacement components $\delta_{x}$ and $\delta_{y}$ in the $x$ - and $y$-directions, respectively, can be evaluated in the whole field of observation. Knowing the local surface normal at Q, the surface slope components in, say, the $x-z$ and $y-z$ planes can be evaluated as [24],

$\phi_{x ; y} \approx \tan \phi_{x ; y}=2 \frac{\partial w}{\partial x ; y}=\frac{\delta_{x ; y}}{\Delta}$

where $\phi_{x ; y}$ represent angular deflections of light rays. Here $\Delta$ denotes the distance between the specimen and target planes and is the optical lever that controls the sensitivity of the method in addition to those associated with digital image correlation such as the sub-image size, pixel overlap and the searching algorithm, to name a few. It should be noted that the coordinates of the target plane should be mapped to that of the specimen plane using $(x ; y)=$ $\frac{L}{L+\Delta}\left(x_{0} ; y_{0}\right)$ where $L$ is the distance between the camera and the specimen and the subscript ' 0 ' denotes coordinates of the target plane [22]. A detailed set of baseline experiments including the role of target plane speckle characteristics, specimen-to-target plane distance, sub-image size used for during image correlation, to name a few, can be found in Refs. [25, 26].

\section{Quasi-Static Experiments: Mode-I and Mixed- Mode}

Quasi-static 3-point bend experiments were performed on edge notched PMMA specimens using r-DGS. A $130 \times 60 \times 8.9 \mathrm{~mm}^{3}$ specimen was used for both mode-I and mixed-mode loading configurations. An initial crack of length $12 \mathrm{~mm}$ normal to the edge (crack length to plate width ratio $a / W=0.2$ ), as shown in the Fig. $2 \mathrm{a}$, was used for mode-I loading and an initial crack of $12 \mathrm{~mm}$ at $45^{\circ}$ to the edge (Fig. 2b) was used for mixed-mode experiments. The specimen resting on two anvils (120 mm span) was loaded in a displacement control mode (cross-head speed $=0.005 \mathrm{~mm} / \mathrm{s}$ ) using Instron 4465 universal testing machine. One of the two $130 \times 60 \mathrm{~mm}^{2}$ faces of the specimen was deposited with a thin aluminum film to make the surface specularly reflective. A 50/50 beam splitter was positioned between the specimen and the camera at an angle of $45^{\circ}$ to the optical axis of the camera (see Fig. 3). A speckle target plate, sprayed with alternate mists of black and white paint to create a random speckle pattern, was placed at $45^{\circ}$ to the beam splitter.

The normal distance between the specimen surface and the speckle plane was $(\Delta=) 65 \mathrm{~mm}$. In this optical arrangement, the camera was focused on the speckles via
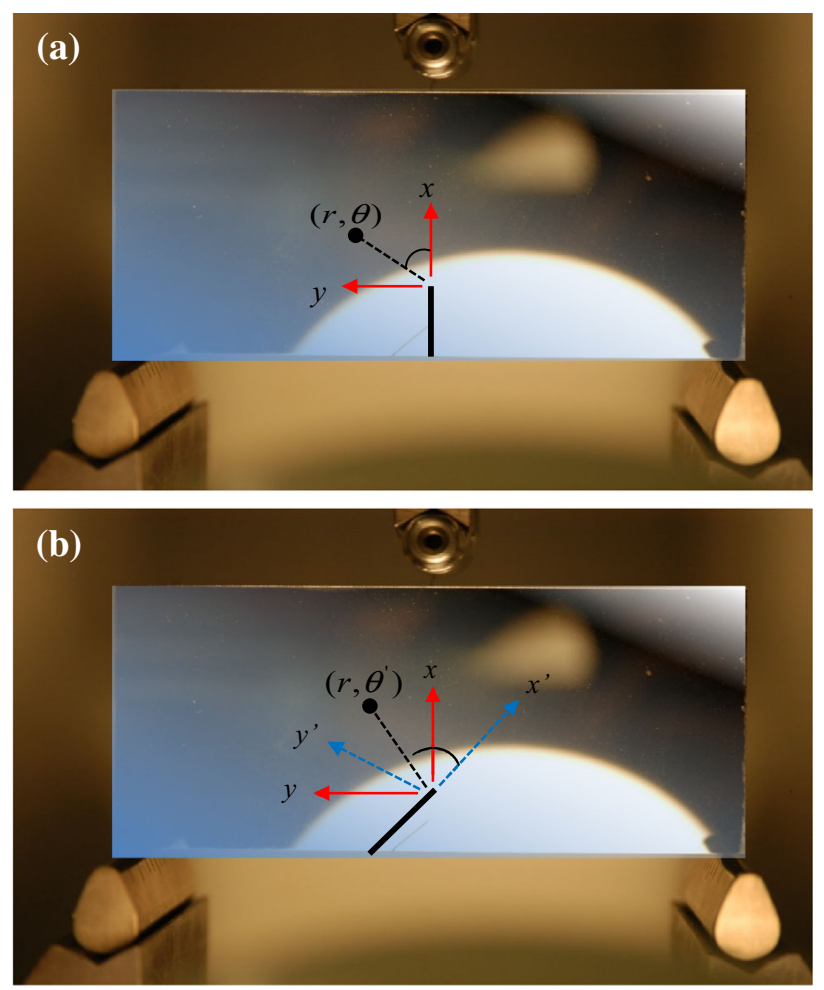

Fig. 2 Specimen configurations for crack tip deformation measurement using r-DGS: a mode-I loading, b mixed-mode-I/II loading. Reflections of extraneous objects due to specular nature of the specimen are also evident
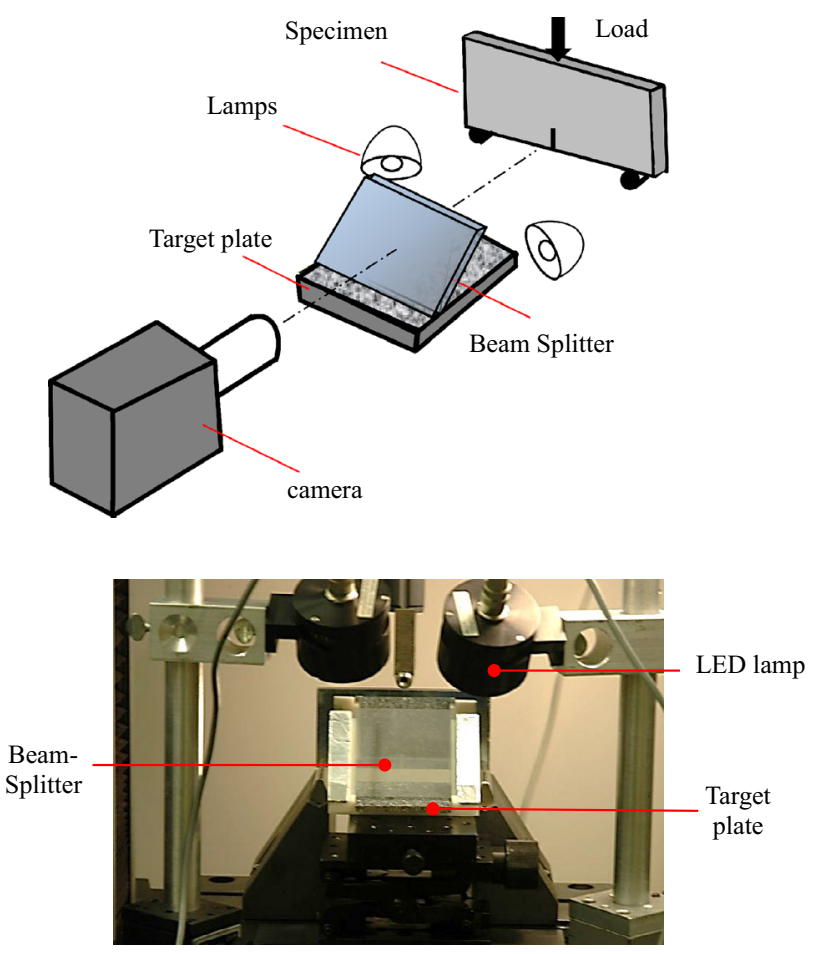

Fig. 3 Schematic of the experimental setup to measure crack-tip deformations (top). Photograph (front view) of the experimental setup (bottom) 
Fig. 4 Representative speckle images recorded during quasistatic tests using r-DGS setup. Mode I image covers $\left(42 \times 28 \mathrm{~mm}^{2}\right)$, and mixedmode image covers $\left(46.5 \times 31 \mathrm{~mm}^{2}\right)$
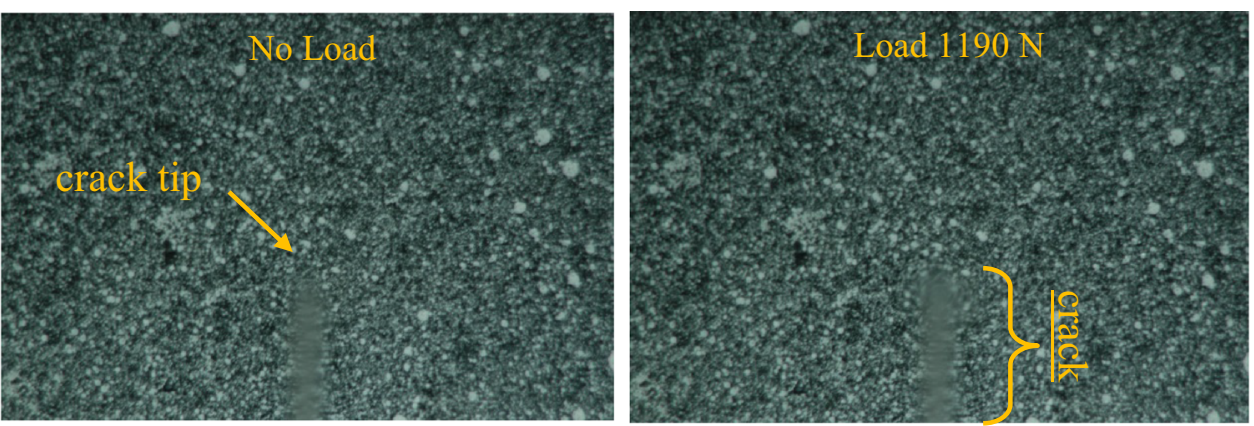

Mode-I
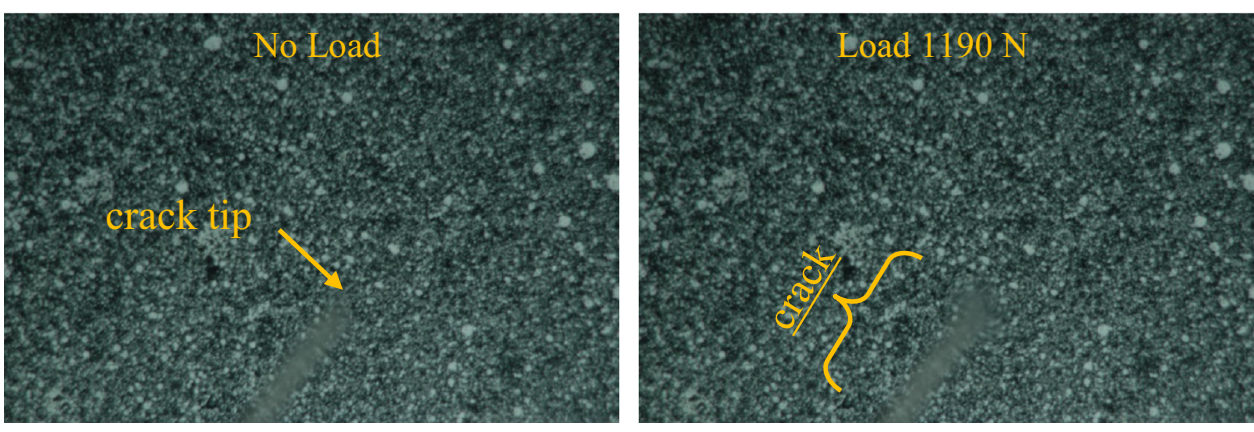

Mixed-Mode-I/II the specular specimen surface. The target plate was marked with a few reference points to obtain the scale factor for relating the image dimensions with the actual specimen dimensions. A Nikon D100 digital SLR camera fitted with 28-300 mm focal length macro lens and an adjustable bellows was placed in front of the specimen at a distance of $\sim 1150 \mathrm{~mm}$. A small aperture $\left(F^{\#}=32\right)$ was selected for achieving a good depth-of-focus. The target plate was uniformly illuminated by a pair of LED lamps. A reference image was recorded with a camera resolution of $1504 \times 1000$ pixels (one pixel covered $28.06 \mu \mathrm{m}$ on the target for mode-I experiments and $31.06 \mu \mathrm{m}$ for mixedmode experiments) in no-load/reference state. During the experiment, speckle images were recorded once every $5 \mathrm{~s}$ and at different load levels representing deformed states of the specimen. A pair of speckle images corresponding to the reference and deformed states for mode-I and mixedmode cases are shown in Fig. 4. In these images the crack can be seen as a smeared gray line normal to or inclined to the lower edge of the image.

Due to deformations in the crack-tip vicinity, the reflected light rays carry surface slope information relative to the reference state. Using 2-D DIC, the angular deflection fields $\phi_{x}$ and $\phi_{y}$ were obtained by correlating images corresponding to the deformed state with the one from the reference state. The images were divided into $15 \times 15$ pixel sub-images and correlated with a 5 pixel overlap during image correlation. This resulted in an array of
$297 \times 196$ data points around the crack-tip for subsequent extraction of fracture parameters.

Figure 5 shows the resulting surface slopes near the crack-tip for different load levels. The contours represent surface slopes $\frac{\partial w}{\partial x}$ (column-1) and $\frac{\partial w}{\partial y}($ column-2) where $w$ is the displacement in the out-of-plane (in the $z$-) direction. Symmetry and antisymmetry of $\frac{\partial w}{\partial x}$ and $\frac{\partial w}{\partial y}$ contours, respectively, relative to the crack line ( $x$-axis) can be seen for all load levels. The contour lines adjacent to the crack faces appear perturbed due to unavoidable edge effects and finite sub-image size. Further, these contours show qualitative similarities with the reflection-mode CGS counterparts [1517] which measures the same field quantity but one component at a time and as an analog light intensity field.

\section{Mode-I Stress Intensity Factors}

Using Williams' asymptotic stress field expansion for modeI cracks, the in-plane gradients of out-of-plane displacement (or the angular deflection) can be expressed [27] as,

$\frac{\phi_{x ; y}}{2}=\frac{\partial w}{\partial x ; y}=-\frac{v B}{2 E} \sum_{N=1}^{\infty} A_{N}\left(\frac{N}{2}-1\right) r^{\left(\frac{N}{2}-2\right)} \cos \left(\frac{N}{2}-2\right) \theta$

where $E$ is the elastic modulus ( $=3$ GPa for PMMA), $v$ the Poisson's ratio $(=0.35)$, and $B$ the sample thickness 


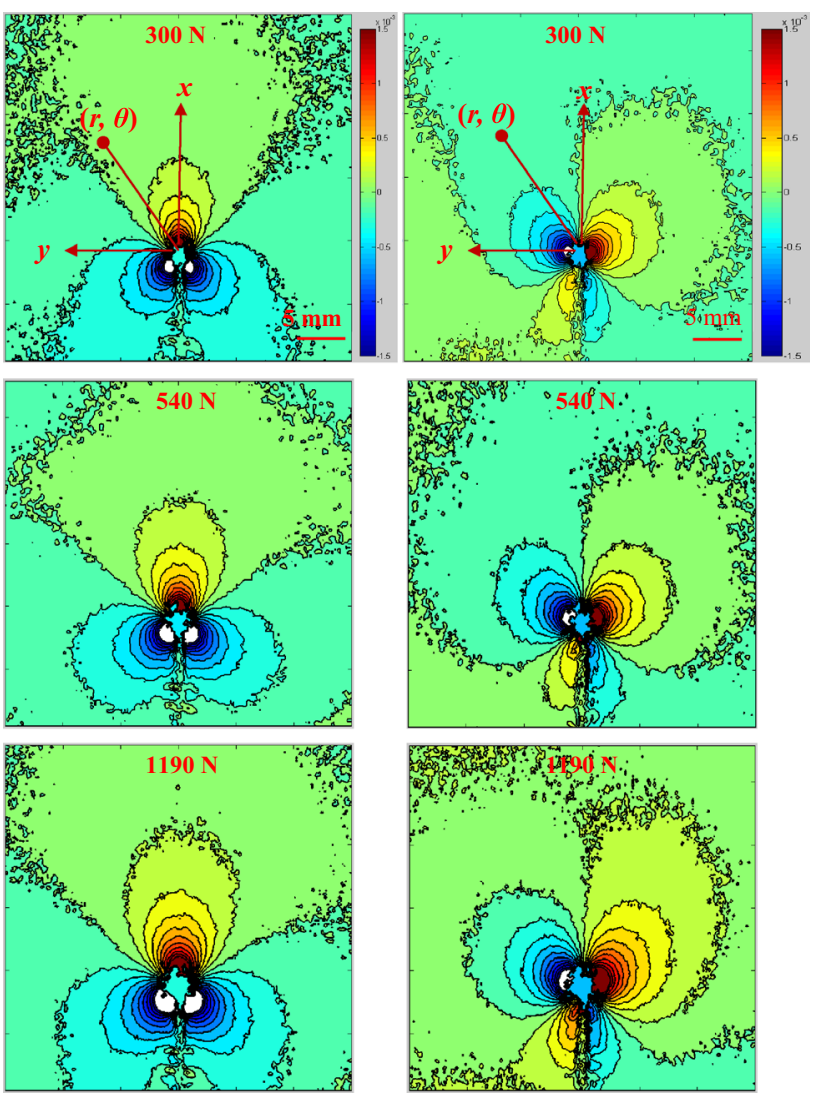

Fig. 5 Experimentally measured contours of angular deflections of light rays $\phi_{x}$ (column-1) and $\phi_{y}$ (column-2) in the crack tip vicinity for different load levels. Contours are plotted in $15 \times 10^{-5}$ radian increments

(=8.9 mm). In the above equation $(r, \theta)$ are the crack-tip polar coordinates and $A_{1}=K_{I} \sqrt{\frac{2}{\pi}}$ with $K_{I}$ being the mode-I stress intensity factor. In Eq. (2), if $K$-dominance is assumed to prevail in the crack-tip vicinity, ${ }^{1}$ terms corresponding to $N>1$ can be neglected to get,

$\frac{\phi_{x ; y}}{2}=\frac{\partial w}{\partial x ; y}=-\frac{v B}{2 E}\left[\left(-\frac{A_{1}}{2}\right) r^{\left(-\frac{3}{2}\right)} \underset{\sin }{\cos }\left(-\frac{3 \theta}{2}\right)\right]$.

An overdeterministic linear regression analysis was performed from the measured angular deflection data based on the estimated crack tip location guided by the tri-lobed contours of surface slopes around the singularity. The angular deflections in the region around the crack-tip $\left(0.5 \leq r / B \leq 1.5,-150^{\circ} \leq \theta \leq+150^{\circ}\right)$ were used in the regression analysis. This region was chosen to minimize the triaxial effects near the crack-tip [10] and to limit analysis to regions rich in $K$-dominant data based on prior knowledge on these field quantities [15]. The variation of

\footnotetext{
1 The specimen geometry used for demonstrating mode-I crack tip deformations is known to produce $K$-dominant deformations as shown in Ref. [15].
}

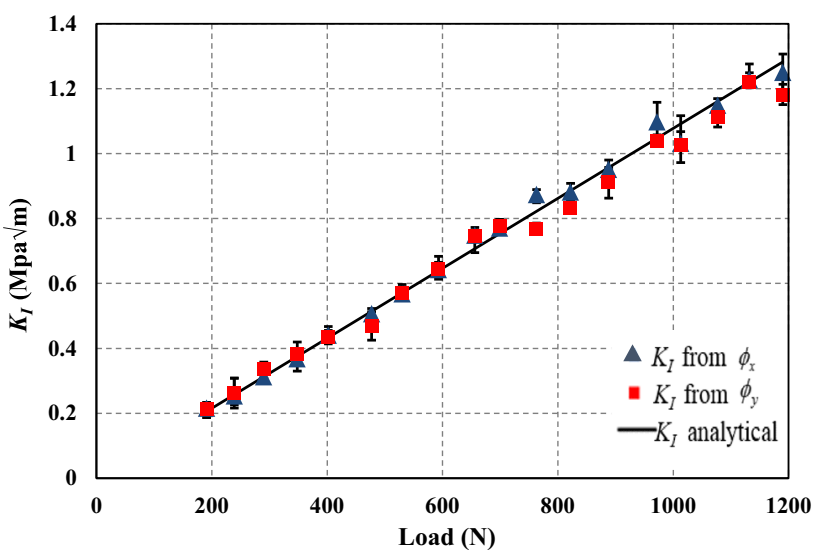

Fig. 6 Variation of experimentally measured mode-I stress intensity factors (symbols) with applied load. The solid line represents stress intensity factors from boundary collocation solution for a static 3-point bend crack problem

stress intensity factors for different load levels are plotted in Fig. 6. The error bars correspond to uncertainty in cracktip location during regression analysis of measured data.

The mode I stress intensity factors based on boundary collocation method is given by [28],

$$
\begin{aligned}
K_{I}= & \frac{F \cdot S}{B \cdot W^{\frac{3}{2}}} \\
& \cdot \frac{3 \lambda^{\frac{1}{2}}\left[1.99-\lambda(1-\lambda)\left\{2.15-3.93 \lambda+2.7 \lambda^{2}\right\}\right]}{2(1+2 \lambda)(1-\lambda)^{\frac{3}{2}}} ; \\
\lambda= & \left(\frac{a}{W}\right),
\end{aligned}
$$

where $F$ is the applied load, $S$ is the distance between the supports, $a$ is the initial crack length, and $W$ is the specimen width. The stress intensity factors obtained from the above equation are plotted in Fig. 6 comparatively with those from the regression analysis of optical data. Evidently the agreement between the analytical and experimental stress intensity factors is good, suggesting r-DGS as a feasible method for mode-I fracture mechanics investigations.

\section{Mixed-Mode Stress Intensity Factors}

Figure 7 shows r-DGS contours due to surface deformations near the mixed-mode crack-tip. The measured orthogonal surface slopes for two select load levels are shown in the global $x$ - and $y$-coordinates defined at the crack-tip and aligned with the loading direction and the specimen edges. The availability of two orthogonal fields offers flexibility to evaluate slopes in a rotated coordinate system aligned with the crack orientation, denoted by the 

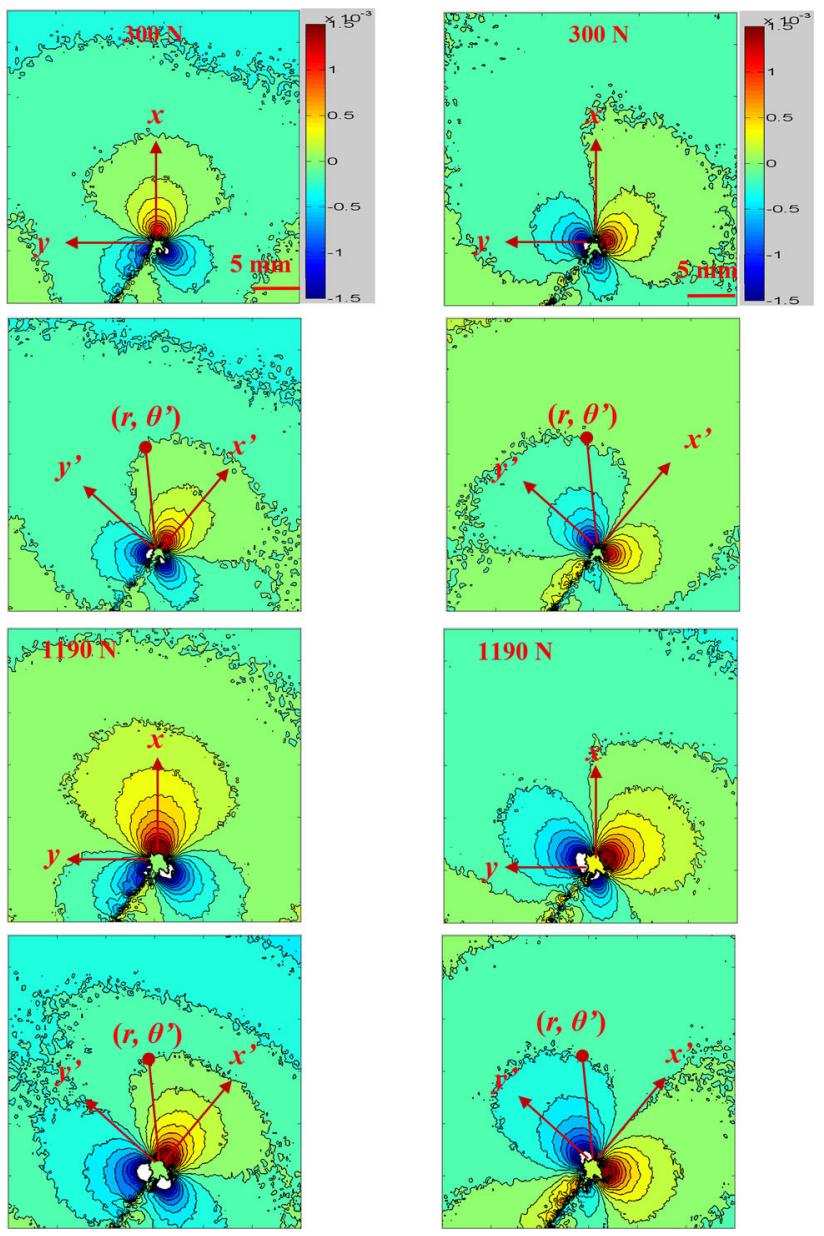

Fig. 7 Contours representing surface slopes in the mixed-mode crack tip vicinity. The contours in 1st and 3rd rows (for two different loads) represent slopes in the global co-ordinates $(x, y)$ and those in 2nd and 4 th rows are in the local coordinates $\left(x^{\prime}, y^{\prime}\right)$. Contour increments are $15 \times 10^{-5}$ radians

coordinates $x^{\prime}$ and $y^{\prime}$ in Fig. 2b. Accordingly, in this research, the orthogonal angular deflections of light rays and hence the respective surface slopes in the local $x^{\prime}$ and $y^{\prime}$ directions were evaluated using transformations,

$\left\{\begin{array}{c}\frac{\phi_{x}^{\prime}}{2} \\ \frac{\phi_{y}^{\prime}}{2}\end{array}\right\}=\left\{\begin{array}{l}\frac{\partial w}{\partial x^{\prime}} \\ \frac{\partial w}{\partial y^{\prime}}\end{array}\right\}=\left(\begin{array}{cc}\cos \alpha & \sin \alpha \\ -\sin \alpha & \cos \alpha\end{array}\right)\left\{\begin{array}{l}\frac{\partial w}{\partial x} \\ \frac{\partial w}{\partial y}\end{array}\right\}$

where $\alpha\left(=45^{\circ}\right.$ in this case $)$ is the crack orientation angle (see, Fig. 2b). Contour plots in Fig. 7 (2nd and 4th rows) represents the surface slope contours after transforming them into the local coordinate system using the corresponding ones in the global coordinates shown in the 1st and 3rd rows, respectively.

The asymptotic expansion for in-plane surface slopes for mixed-mode loading condition is obtained by superposing the mode-I and mode-II fields [27],

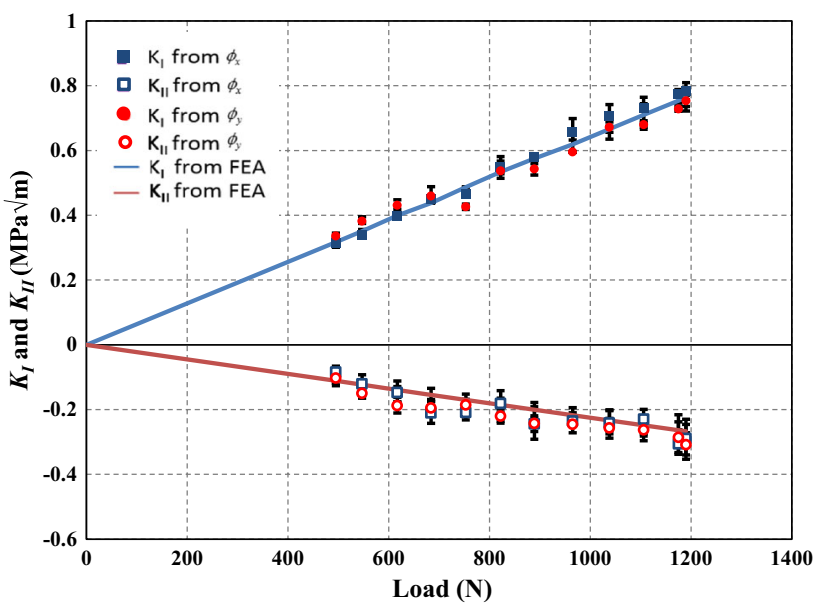

Fig. 8 Comparison of mixed-mode stress intensity factors from r-DGS and finite element simulation

$$
\begin{aligned}
& \frac{\phi_{x}^{\prime}}{2}=\frac{\partial w}{\partial x^{\prime}}=\frac{-v B}{2 E} \\
& {\left[\begin{array}{c}
\left\{\sum_{N=1}^{\infty}\left(A_{N}\left(\frac{N}{2}-1\right) r^{\left(\left(\frac{N}{2}\right)-2\right)} \cos \left(\frac{N}{2}-2\right) \theta^{\prime}\right)\right\} \\
+\left\{\sum_{N=1}^{\infty}\left(B_{N}\left(\frac{N}{2}-1\right) r^{\left(\left(\frac{N}{2}\right)-2\right)} \sin \left(\frac{N}{2}-2\right) \theta^{\prime}\right)\right\}
\end{array}\right]} \\
& \frac{\phi_{y}^{\prime}}{2}=\frac{\partial w}{\partial y^{\prime}}=\frac{-v B}{2 E} \\
& {\left[\begin{array}{r}
\left\{\sum_{N=1}^{\infty}\left(A_{N}\left(\frac{N}{2}-1\right) r^{\left(\left(\frac{N}{2}\right)-2\right)} \sin \left(\frac{N}{2}-2\right) \theta^{\prime}\right)\right\} \\
+\left\{\sum_{N=1}^{\infty}\left(B_{N}\left(\frac{N}{2}-1\right) r^{\left.\left(\frac{N}{2}\right)-2\right)} \cos \left(\frac{N}{2}-2\right) \theta^{\prime}\right)\right\}
\end{array}\right]}
\end{aligned}
$$

where $\left(r, \theta^{\prime}\right)$ are polar coordinates centered at the crack-tip in the local coordinates $x^{\prime}$ and $y^{\prime}$ with $\theta^{\prime}=\theta+\alpha$ ( $\alpha=-45^{\circ}$ is the crack orientation angle), $E$ the elastic modulus, $v$ the Poisson's ratio, $B$ the nominal specimen thickness, $A_{1} ; B_{1}=\sqrt{\frac{2}{\pi}}\left(K_{I} ; K_{I I}\right)$ with $K_{I}$ and $K_{I I}$ being the mode-I and mode-II stress intensity factors, respectively. Solutions based on $N$ up to 3 were used to extract the stress intensity factors. The results thus obtained are plotted in Fig. 8 for different load levels. Again, the error bars are due to variation in locating the crack-tip and hence the values of $\left(r, \theta^{\prime}\right)$ used in the regression analysis.

A finite element analysis was carried out using ABAQUS $^{\text {TM }}$ software package to complement mixed-mode stress intensity factors obtained experimentally as this method has not been implemented previously for mixedmode conditions. The numerical model shown in Fig. 9 
Fig. 9 Finite element model for mixed-mode crack tip analysis. Enlarged view of the mesh near the crack tip is also shown

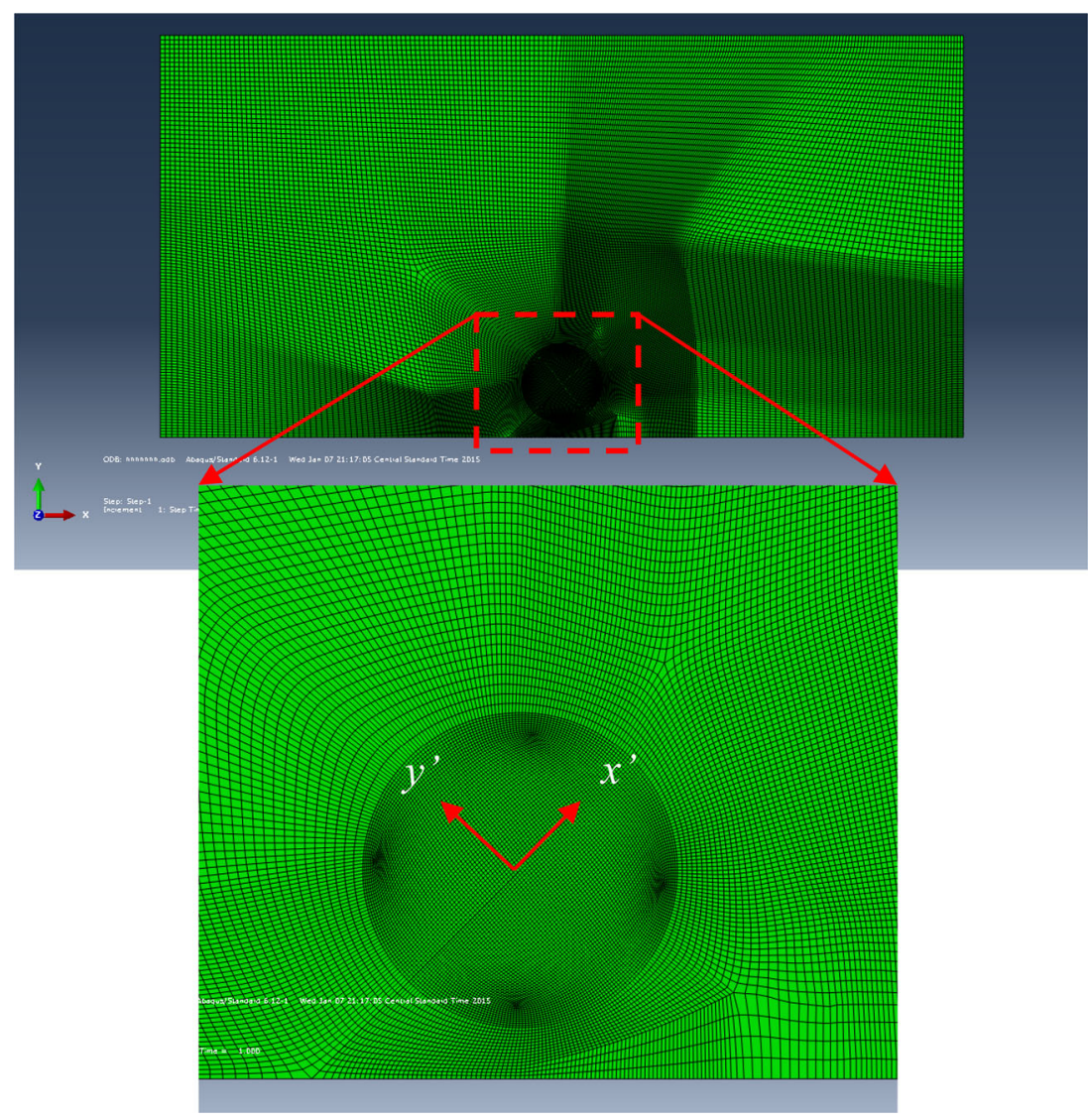

\section{Dynamic Experiments}

crack-tip region was discretized using a fine mesh with the crack-tip element size as small as $0.01 a$ ( $a$ crack length). The mesh near the crack-tip is as shown in the inset of Fig. 9. No attempt was made to create the theoretical $r^{-1 / 2}$ singularity at the crack-tip by incorporating special elements. The elastic constants used in the analysis were the same ones as in experiments. The crack opening and crack sliding displacements from the finite element simulations were used to obtain the two stress intensity factors $K_{I}$ and $K_{I I}$. That is, the displacements for the nodes along the two crack flanks were output in the local $x^{\prime}$ and $y^{\prime}$ coordinates. Using these, apparent mode-I and -II stress intensity factors were calculated at each nodal location using,

$\left(K_{I ; I I}\right)_{\text {apparent }}=\left.\frac{E \sqrt{2 \pi}}{8 \sqrt{r}}\left(u_{2 ; 1-\text { upper }}-u_{2 ; 1-\text { lower }}\right)\right|_{(r, \theta= \pm \pi)}$

where $u_{2}$ is the crack face opening displacement and $u_{1}$ is crack face sliding displacement at a node along the upper and lower crack faces. The linear region of apparent $K_{I}$ and $K_{I I}$ extrapolated to the crack-tip provided the two stress intensity factors. Figure 8 shows the variation of these computations overlaid on experimental results. A good match between the two is evident from the plot, suggesting the feasibility of r-DGS for studying mixed-mode fracture problems.
The crack-tip deformation measurements under mode I and mixed-mode (I/II) conditions for an edge notched PMMA specimen during stress wave propagation were performed using a long-bar impactor in conjunction with high-speed photography and r-DGS method. The schematic of the experimental setup is as shown in Fig. 10. The loading device consisted of an aluminium 7075-T6 long-bar (25.4 mm diameter and $2 \mathrm{~m}$ long) with a cylindrical impacting head, a gas-gun and a high-speed digital camera. The long-bar was aligned coaxially with the barrel of the gas-gun housing a $305 \mathrm{~mm}$ long, $25.4 \mathrm{~mm}$ diameter aluminum striker. The crack-tip deformations were photographed using a Cordin 550 high-speed digital camera equipped with $32 \mathrm{CCD}$ sensors and two highenergy flash lamps to illuminate the target plate. A 50/50 beam splitter positioned between the lens and the specimen at $45^{\circ}$ angle was used to view the speckle pattern on the target via the reflective face of the specimen. Other experimental parameters such as the framing rate, flash duration and image storage were managed using a computer connected to the camera. The cylindrical tip of the long-bar was registered against the notchfree edge of the specimen (of dimensions $130 \times 60 \times$ $8.9 \mathrm{~mm}^{3}$ ) in both mode-I and mixed-mode loading experiments. The specimens were rested on an adjustable platform 
Fig. 10 Schematic of the experimental setup used in the dynamic fracture study. Inset is the photograph showing the close-up view of the optical arrangement in front of the sample

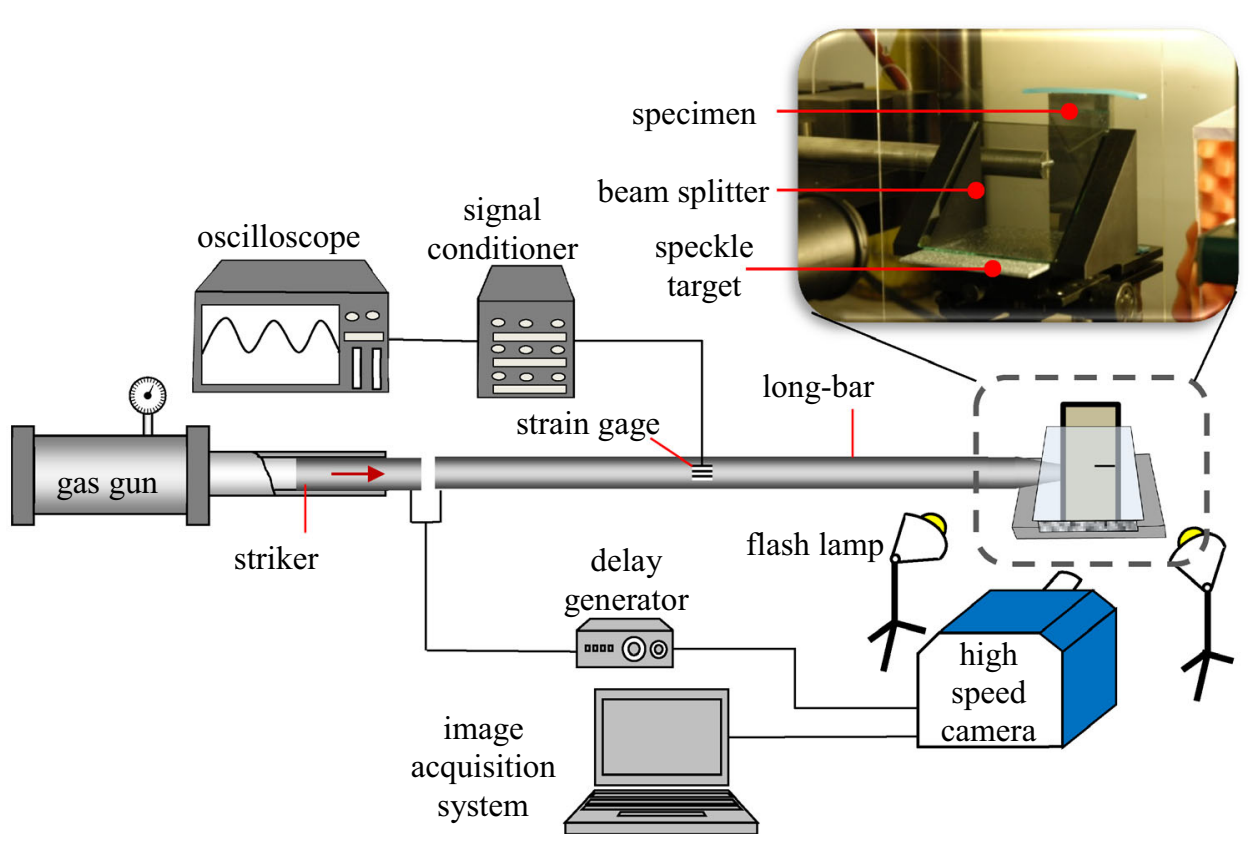

and sandwiched between two strips of soft putty ( $2 \mathrm{~mm}$ thick) at the top and bottom faces $\left(60 \times 8.9 \mathrm{~mm}^{2}\right)$ to simulate 'freefree' conditions along those faces. For mode-I loading the tip of the long-bar was in-line with the edge notch whereas for the mixed-mode loading experiments an eccentricity of $20 \mathrm{~mm}$ with respect to the crack line and the axis of the long-bar (see, Fig. 11) was used. As in the quasi-static experiments, one of the faces of the specimen $\left(130 \times 60 \mathrm{~mm}^{2}\right)$ was made specularly reflective by sputter coating it with aluminum. The dynamic loading was initiated by suddenly discharging compressed air from the gas-gun chamber which propelled the striker situated in the barrel. A compressive stress wave was initiated in the long-bar when the striker impacted the longbar, and the resulting stress waves travelled the length of the bar before imparting a transient line-load to the edge of the specimen at the desired location.

A strain gage (CAE-13-125UN-120, Micro-Measurements Group) bonded to the long-bar was used to measure the strain history in the bar during impact loading. The measurements were performed using a Lecroy oscilloscope and an Ectron signal amplifier/conditioner with a Wheatstone quarter-bridge circuit. As soon as the striker contacted the long-bar, an electrical circuit was completed. Using a pre-set delay on a delay generator, the high-speed camera was triggered to photograph speckles on the target plane via the specimen surface during stress wave propagation. Particle velocity of the long-bar was subsequently calculated using the strain history measured from the oscilloscope. In these experiments, the distance between the target plate and specimen was $(\Delta=) 102 \mathrm{~mm}$ (and the distance between the camera lens and the specimen was $\sim 715 \mathrm{~mm}$ ). impact

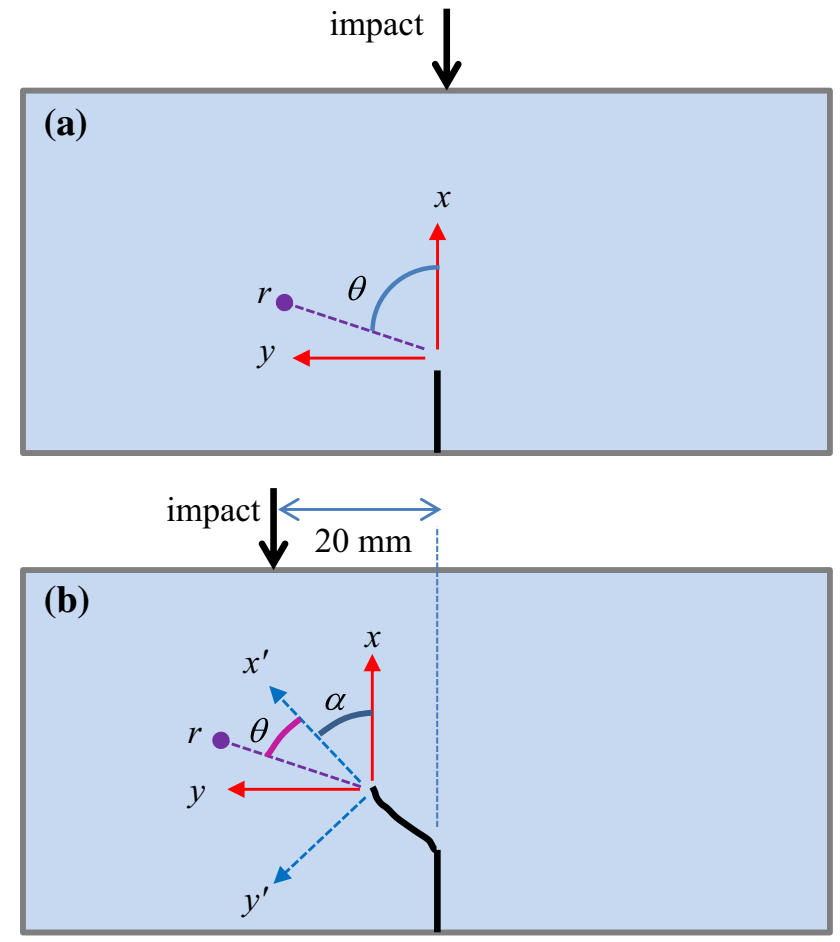

Fig. 11 Specimen loading configuration and crack tip coordinate system used in dynamic fracture experiments. a Global crack tip coordinates (mode-I), b global and local coordinates for mixed-mode crack propagation. Specimen size $=130 \times 60 \mathrm{~mm}^{2}$

To carry out digital image correlation, a set of 32 reference images (one image from each CCD sensor of the high-speed camera) prior to loading was captured by operating the camera at 150,000 frames per second. Next 

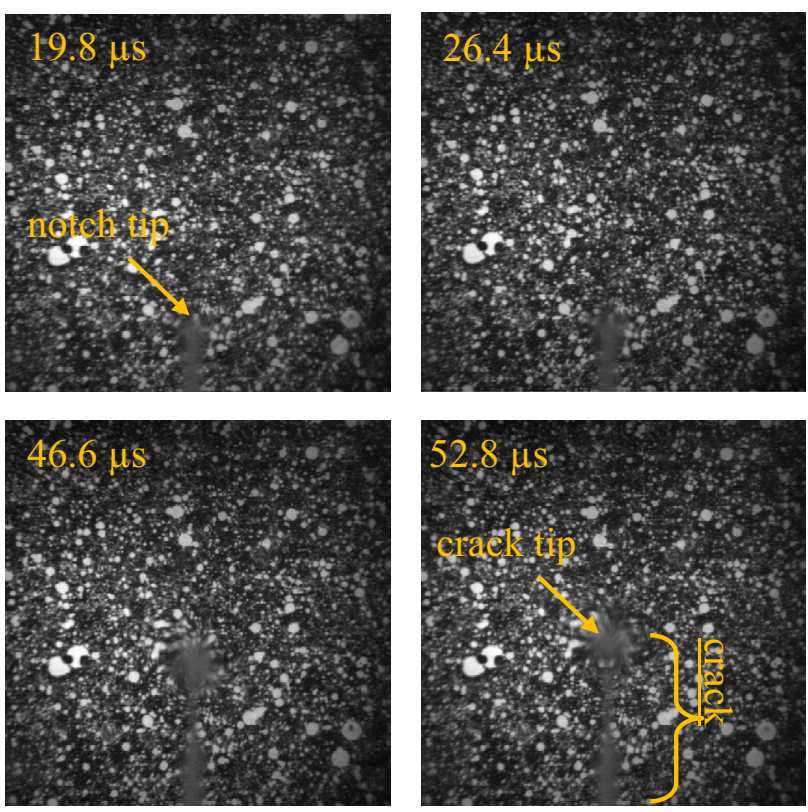

Fig. 12 Speckle images captured for mode-I loading. Defocussed vertical line corresponds to the edge crack; stationary but dynamically loaded (row-1) and dynamically growing (row-2) (Image covers $36.1 \times 36.1 \mathrm{~mm}^{2}$ )

the camera was triggered as the striker contacted the longbar. A second set of 32 speckle images was captured at the same framing rate while the specimen was experiencing transient loading. A set of speckle images recorded during mode-I and mixed-mode dynamic fracture experiment is shown in Figs. 12 and 13. These images show speckle recordings corresponding to a few select time instants before and after crack initiation. In these, the current location of the crack-tip can be seen as the terminus of a smeared strip of speckles.

The deformed and reference image pairs recorded by the same sensor of the high-speed camera were paired and correlated to obtain orthogonal displacements $\delta_{x}$ and $\delta_{y}$ on the target plane (see, Fig. 1). These were subsequently converted into orthogonal angular deflections of light rays $\phi_{x}$ and $\phi_{y}$ in the $x-z$ and $y-z$ planes, respectively, and the corresponding surface slopes $\frac{\partial w}{\partial x}$ and $\frac{\partial w}{\partial y}$ on the specimen plane. The image correlation was carried out using a sub-image size of $15 \times 15$ pixels (one pixel covered $36.1 \mu \mathrm{m}$ on the target for mode-I experiments and $34.8 \mu \mathrm{m}$ for mixed-mode experiments) with 5 pixels overlap during analysis.

\section{Dynamic Results: Mode-I}

Figure 14 shows surface slopes around dynamically loaded mode-I crack. The first two rows of images correspond to the pre-crack initiation and the last two to the post-crack initiation regimes. Following crack initiation, it can be seen
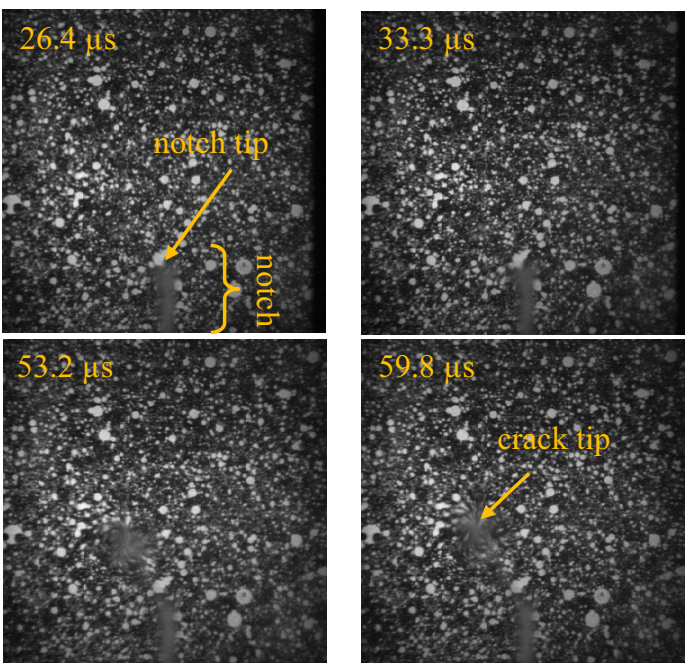

Fig. 13 Speckle images captured for mixed-mode (mode-I/II) loading: First row corresponds to before crack initiation and the second row for a kinked growing crack relative to the initial vertical notch $\left(\right.$ area shown $\left.=34.8 \times 34.8 \mathrm{~mm}^{2}\right)$

that the crack travels self-similarly in the direction of the initial notch without any kink due to the imposed symmetric loading. These contours show qualitative similarity with those for the static mode-I results shown in Fig. 5. The contours immediately adjacent to the crack-tip are lost due to a combination of diffraction effects, the numerical aperture of the high-speed camera used and the severity of deformations limiting the correlation of speckles. This however is comparable to similar issues associated with the formation of a shadow spot or sheared images in photoelastic and coherent gradient sensing counterparts.

The expressions for surface slopes corresponding to a steadily propagating crack in an elastic planar solid are given by [27],

$$
\begin{aligned}
& \frac{\phi_{x}(t)}{2}=\frac{\partial w(t)}{\partial x}=\frac{-v B}{2 E} \\
& {\left[\begin{array}{r}
f\left(V ; C_{L}, C_{S}\right) \\
\left\{\left(A_{1}(t)\left(\frac{N}{2}-1\right) r\left(\left(\frac{N}{2}\right)-2\right) \cos \left(\frac{N}{2}-2\right) \theta\right)\right\} \\
\left.+\sum_{N=2}^{\infty}\left(A_{N}(t)\left(\frac{N}{2}-1\right) r\left(\left(\frac{N}{2}\right)-2\right) \cos \left(\frac{N}{2}-2\right) \theta\right)\right]
\end{array}\right.}
\end{aligned}
$$

$$
\begin{aligned}
& \frac{\phi_{y}(t)}{2}=\frac{\partial w(t)}{\partial y}=\frac{-v B}{2 E} \\
& {\left[\begin{array}{r}
f\left(V ; C_{L}, C_{S}\right) \\
\left\{\left(A_{1}(t)\left(\frac{N}{2}-1\right) r\left(\left(\frac{N}{2}\right)-2\right) \sin \left(\frac{N}{2}-2\right) \theta\right)\right\} \\
\left.+\sum_{N=2}^{\infty}\left(A_{N}(t)\left(\frac{N}{2}-1\right) r\left(\left(\frac{N}{2}\right)-2\right) \sin \left(\frac{N}{2}-2\right) \theta\right)\right]
\end{array}\right.}
\end{aligned}
$$




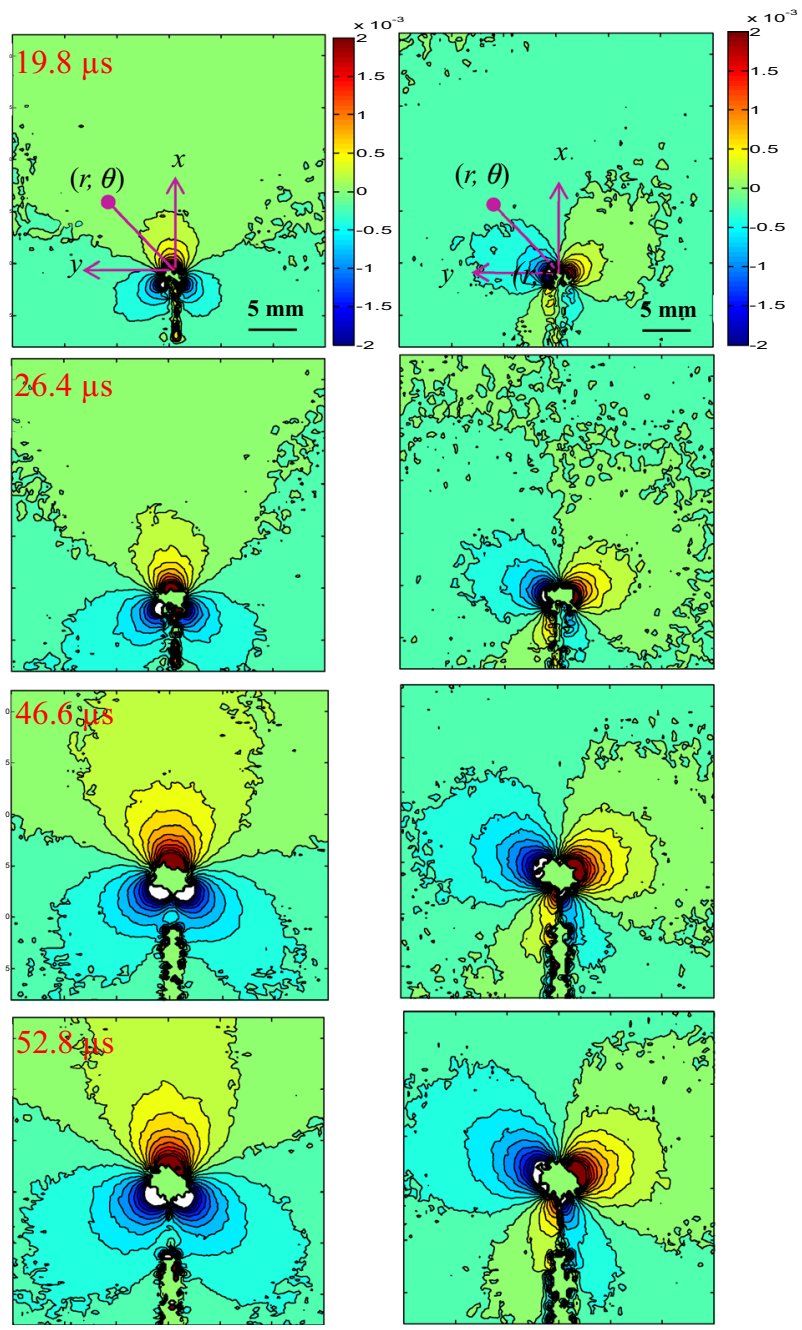

Fig. 14 Experimentally measured $\phi_{x}$ (column 1) and $\phi_{y}$ (column 2) contours near the mode-I crack-tip at different time instants during dynamic impact. Contours are plotted in increments of $2 \times 10^{-4}$ radians. Time $t=0$ corresponds to instant just before crack tip deformation is evident when deformations are contoured

where, $E$ is the elastic modulus ( $=5.8 \mathrm{GPa}$ for $\left.\mathrm{PMMA}^{2}\right), f$ is a function of crack velocity $V$, dilatational and shear wave speeds $C_{L}(=2602 \mathrm{~m} / \mathrm{s})$ and $C_{S}(=1354 \mathrm{~m} / \mathrm{s})$ respectively and $A_{1}(t)=K_{I}(t) \sqrt{\frac{2}{\pi}}$ with $K_{I}(t)$ being the instantaneous mode-I stress intensity factor. (The dilatational and shear wave speeds of the specimen were measured using ultrasonic pulse-echo measurements.) Also, for plane stress conditions,

$f\left(V ; C_{L}, C_{S}\right)=\frac{\left(1+\alpha_{S}^{2}\right)\left(\alpha_{L}^{2}-\alpha_{S}^{2}\right)}{4 \alpha_{S} \alpha_{L}-\left(1+\alpha_{S}^{2}\right)^{2}}$

\footnotetext{
2 Dynamic elastic modulus was based on ultrasonic pulse-echo measurement of longitudinal and shear wave speeds and mass density.
}

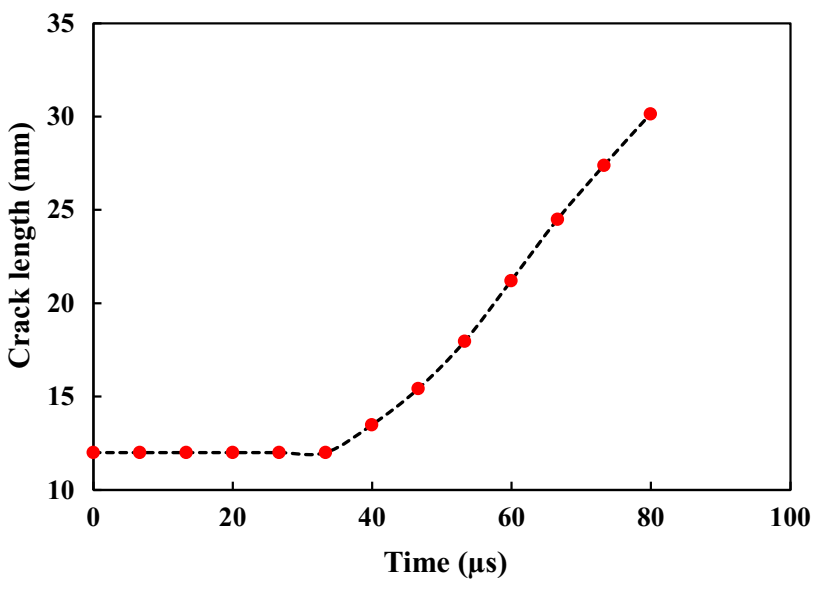

Fig. 15 Crack length history in edge cracked PMMA specimen subjected to mode-I loading

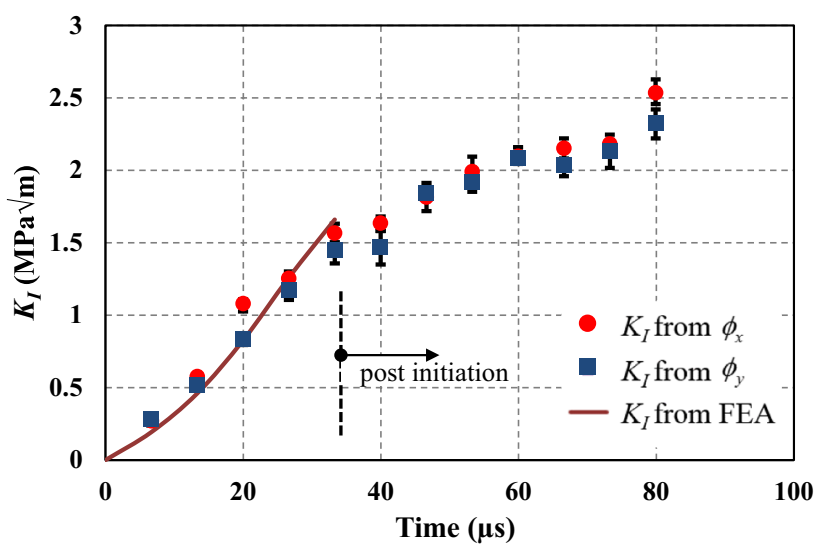

Fig. 16 Dynamic mode-I stress intensity factor histories extracted from surface gradient data. Solid line represents SIF histories from a companion elasto-dynamic finite element analysis based on measured impact velocity input

where, $\alpha_{L: S}=\sqrt{1-\frac{V^{2}}{C_{L: S}^{2}}}$. The crack velocity for the propagating crack was estimated by measuring the change in the crack length between two successive images and dividing by the time interval between them (1/framing rate) and it ranged between 250 and $400 \mathrm{~m} / \mathrm{s}$. The instantaneous crack length measured from the high-speed camera images are shown in Fig. 15. Equations (8) were then used to extract the dynamic mode-I stress intensity factor $\left(K_{I}(t)\right)$ history by performing an overdeterministic analysis of the measured surface slopes in the crack-tip vicinity. A three-term expansion of surface slopes $(N=3$ in Eq. 8) was employed. As in the quasi-static case, data in the $(0.5 \leq r / B \leq 1.5)$ and $(-150 \leq \theta \leq 150)$ range were used in the analysis. The error bars in the Fig. 16 correspond to the variation of stress intensity factor resulting from locating the crack-tip (origin) in the images. Evidently, the crack initiates at about $33.3 \mu$ s after impact 

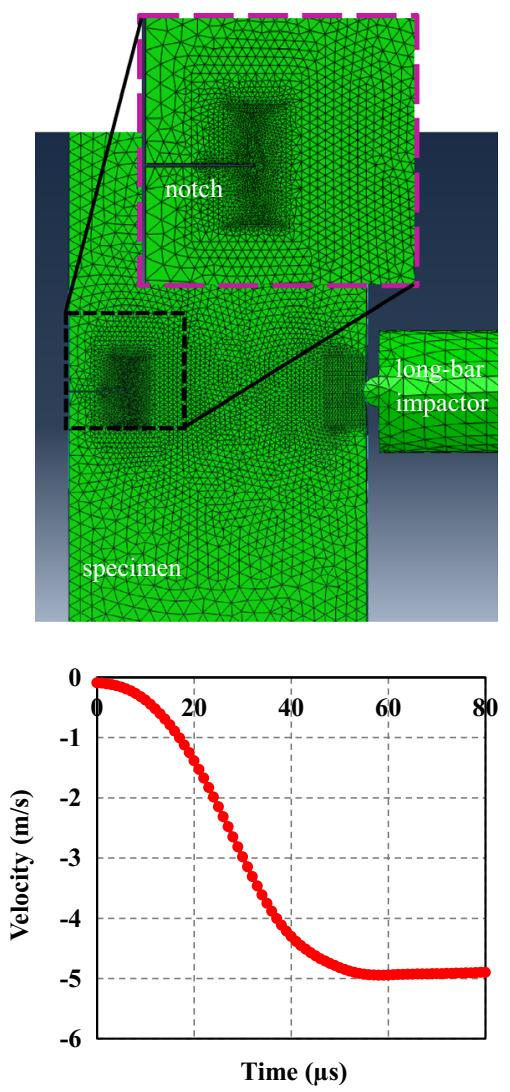

Fig. 17 Finite element model used to simulate dynamic loading (top) and velocity history (bottom) used as an input to the simulation

and the mode-I stress intensity factors increase monotonically during the crack-tip loading phase. Immediately following crack initiation, a visible dip in the stress intensity factor value is evident due to instantaneous unloading of the crack-tip vicinity. Subsequently, the stress intensity factors continue to increase but more modestly showing a tendency to plateau at the later stages of the observation window.

A companion 3-D elasto-dynamic finite element simulation of the mode-I dynamic loading experiment was performed using ABAQUS ${ }^{\mathrm{TM}} /$ Explicit structural analysis software package. The simulation was limited to determining the stress intensity factors independently for a $d y$ namically loaded stationary crack. The model included an edge cracked specimen and a long-bar as shown in Fig. 17. The discretized finite element model consisted of 176,711 quadratic 3D elements $(\mathrm{C} 3 \mathrm{D} 10 \mathrm{M})$ with a fine mesh near the crack-tip having element size of $\sim 0.1 \mathrm{~mm}$. The semicircular head of the long-bar was in contact with the specimen (frictionless contact was defined between the two bodies) along the edge opposite to the crack-tip. The particle velocity measured from the strain history on the bar was input on the far end of the long-bar. The particle velocity $V_{p}$ (Fig. 17) on the long-bar was obtained from,

$V_{p}=C_{A l} \varepsilon$

where $C_{A l}$ is the wave speed of the $\mathrm{Al}$ bar $(=5370 \mathrm{~m} / \mathrm{s})$ and $\varepsilon$ is the measured strain history from the strain gage. The stress waves were transmitted to the specimen from the semicircular head of the long-bar placed against the edge of the specimen. The time steps during the analysis were allowed to be controlled automatically by the explicit integration scheme. The instantaneous mechanical fields including inplane displacements were output at time instants corresponding to the framing rate (or, every $6.6 \mu$ s once) used during experiments. From these displacement fields, the instantaneous mode-I stress intensity factor was calculated using the regression analysis of crack opening displacements adopting the procedure outlined earlier in the quasi-static counterpart since the functional form of the crack-tip fields are the same until crack initiation, with the inertia effects accounted for by the elasto-dynamic computations. The stress intensity factors corresponding to the earliest measurable deformation from the finite element simulation near the crack faces is taken as the first time interval and the instant prior to that was designated $t=0$. The computed data is superimposed with the experimental data up to the crack initiation in the Fig. 16 and a good agreement between experimental and numerical results is readily evident.

\section{Dynamic Results: Mixed-Mode}

Figures 18 and 19 show the evolution of r-DGS contours near a dynamically loaded mixed-mode crack-tip at a few select time instants. The measured orthogonal surface slopes (Fig. 18) are shown using the global crack-tip coordinates $x$ and $y$ (aligned with the loading direction and the specimen edges shown in Fig. 11) for time instants before crack initiation. The contour plots of surfaces slopes in Fig. 19 represent the ones for the post-initiation regime following crack kinking from its initial orientation. Here Fig. 19a, c show the surface slopes in the global coordinates whereas the same have been transformed to the local coordinates and shown in Fig. 19b, d. As in the quasi-static counterparts, a rotation of the contour lobes as a whole after coordinate transformation is evident.

The stress intensity factors in the pre-crack initiation phase was performed in the global coordinate system ( $x$ and $y$ ) defined at the crack-tip aligned with the loading direction and the specimen edges (Fig. 10). The stress intensity factors in the pre-crack initiation regime for a stationary but dynamically loaded crack was evaluated using the mixed-mode asymptotic equation [27], 


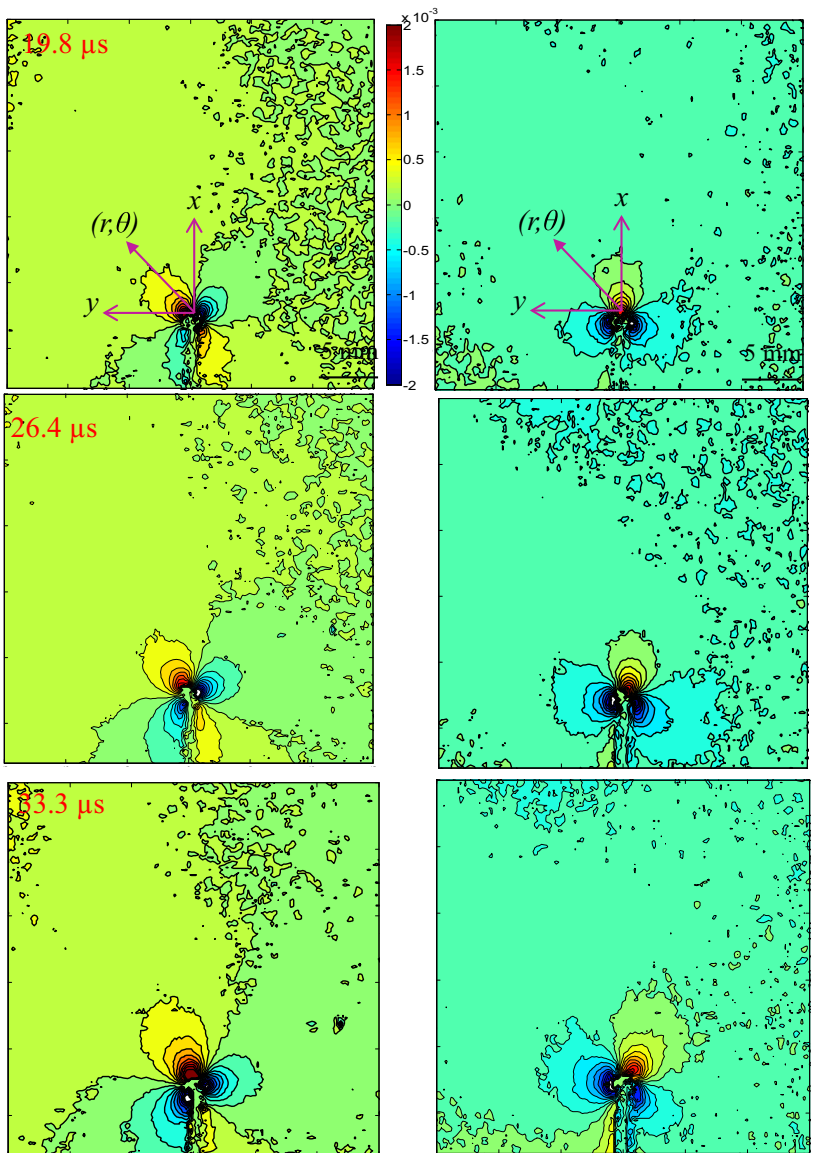

Fig. 18 Experimental $\phi_{x}$ (column 1) and $\phi_{y}$ (column 2) contours near the crack-tip before crack initiation. Contours are plotted every $20 \times 10^{-5}$ radians (a)

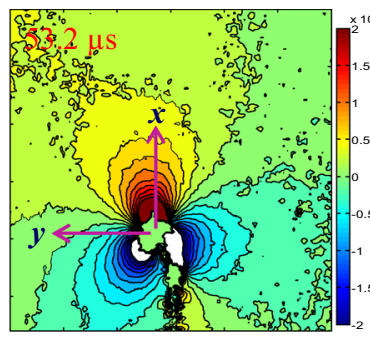

(b)

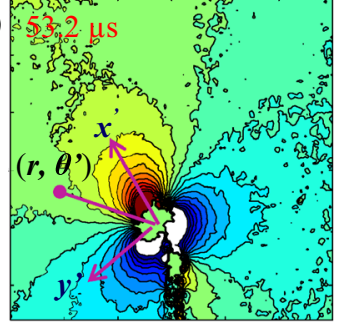

(c)

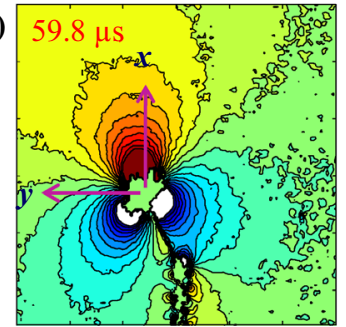

(d)

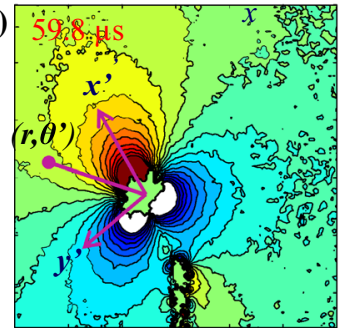

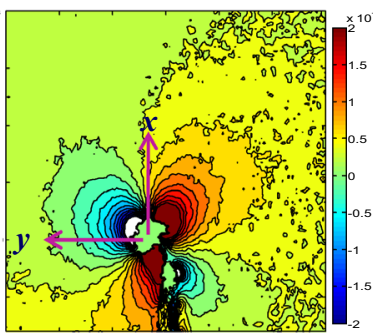
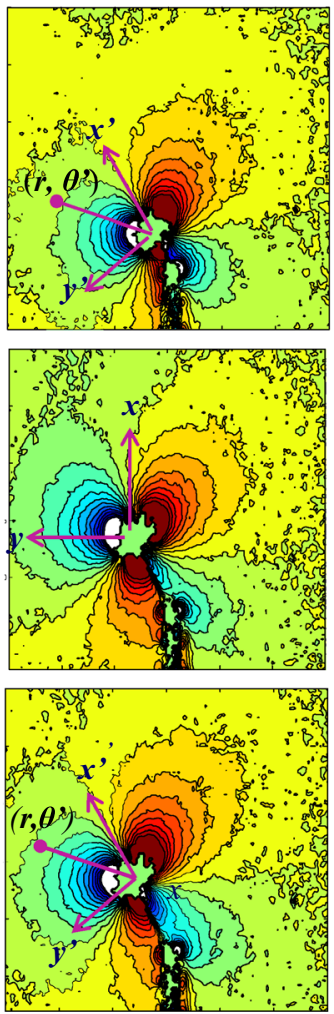

Fig. 19 Experimental $\phi_{x}$ (column 1) and $\phi_{y}$ (column 2) contours near the crack-tip in global coordinates $(\mathbf{a}, \mathbf{c})$ and local coordinates $(\mathbf{b}$, d). Contours increments $20 \times 10^{-5}$ radians

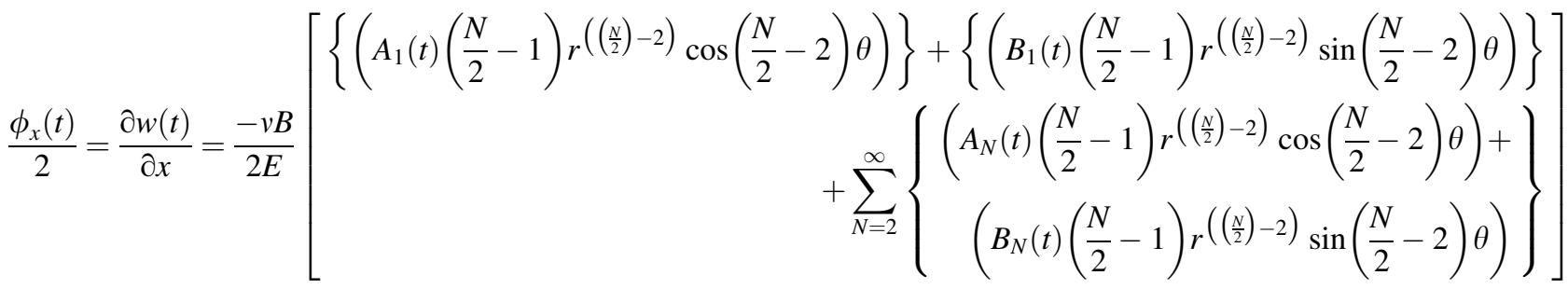

where $(r, \theta)$ are polar coordinates centered at the crack-tip in the global coordinates and all others as defined previously.

The evaluation of stress intensity factors in the postcrack initiation regime was performed by re-orienting the surface slopes in the instantaneous crack propagation direction. That is, along the local coordinates $x^{\prime}$ and $y^{\prime}$ as shown Fig. 19. The orthogonal angular deflections of light rays and hence the respective surface slopes in the $x^{\prime}$ and $y^{\prime}$ directions were evaluated using the transformation, 


$$
\begin{gathered}
\frac{\phi_{x}^{\prime}(t)}{2}=\frac{\partial w(t)}{\partial x^{\prime}}=\frac{\partial w(t)}{\partial x} \cos \alpha(t)+\frac{\partial w(t)}{\partial y} \sin \alpha(t) \\
\frac{\phi_{y}^{\prime}(t)}{2}=\frac{\partial w(t)}{\partial y^{\prime}}=-\frac{\partial w(t)}{\partial y} \sin \alpha(t)+\frac{\partial w(t)}{\partial x} \cos \alpha(t)
\end{gathered}
$$

where $\alpha(t)$ is the instantaneous crack orientation angle. Examples of surface slope contours using this transformation are shown in Fig. 19b, d for time instants 53.2 and $59.8 \mu \mathrm{s}$, respectively. The stress intensity factors were evaluated by using steady-state approximation, where $\alpha_{L: S}=\sqrt{1-\frac{V^{2}}{C_{L: S}^{2}}}$. The instantaneous crack velocity $V$ for the propagating crack was calculated by knowing the change in the crack length (see, Fig. 20) between two successive images and was found to be in the $150-250 \mathrm{~m} / \mathrm{s}$ range, lower than the mode-I counterpart. Again, a threeterm expansion was used to extract the stress intensity factors and was found to capture the overall behavior of a mixed-mode crack. The least-squares analysis was performed on the data in the range $(0.5 \leq r / B \leq 1.5)$ and

$\frac{\phi_{x}^{\prime}(t)}{2}=\frac{\partial w(t)}{\partial x^{\prime}}=\frac{-v B}{2 E}\left[\begin{array}{r}f\left(V ; C_{L}, C_{S}\right)\left\{\left(A_{1}(t)\left(\frac{N}{2}-1\right) r\left(\left(\frac{N}{2}\right)-2\right) \cos \left(\frac{N}{2}-2\right) \theta^{\prime}\right)\right\} \\ +g\left(V ; C_{L}, C_{S}\right)\left\{\left(B_{1}(t)\left(\frac{N}{2}-1\right) r\left(\left(\frac{N}{2}\right)-2\right) \sin \left(\frac{N}{2}-2\right) \theta^{\prime}\right)\right\} \\ +\sum_{N=2}^{\infty}\left[\begin{array}{r}\left(A_{N}(t)\left(\frac{N}{2}-1\right) r\left(\left(\frac{N}{2}\right)-2\right) \cos \left(\frac{N}{2}-2\right) \theta^{\prime}\right)+ \\ \left(B_{N}(t)\left(\frac{N}{2}-1\right) r\left(\left(\frac{N}{2}\right)-2\right) \sin \left(\frac{N}{2}-2\right) \theta^{\prime}\right)\end{array}\right]\end{array}\right]$

where $f$ and $g$ denote functions associated with the instantaneous crack velocity, dilatational and shear wave speeds $C_{L}$ and $C_{S}$ respectively, and $\left(r, \theta^{\prime}\right)$ denote the cracktip polar coordinates $\left(\theta^{\prime}=\theta+\alpha\right)$ associated with the growing crack. For plane stress, the functions $f$ and $g$ are,

$f\left(V ; C_{L}, C_{S}\right)=\frac{\left(1+\alpha_{S}^{2}\right)\left(\alpha_{L}^{2}-\alpha_{S}^{2}\right)}{4 \alpha_{S} \alpha_{L}-\left(1+\alpha_{S}^{2}\right)^{2}}$

$g\left(V ; C_{L}, C_{S}\right)=\frac{2 \alpha_{S}\left(1-\alpha_{L}^{2}\right)}{4 \alpha_{S} \alpha_{L}-\left(1+\alpha_{S}^{2}\right)^{2}}$

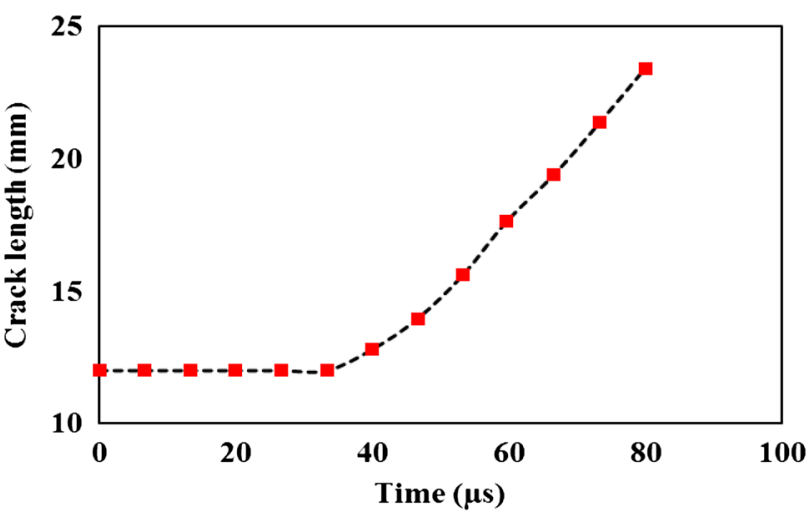

Fig. 20 Dynamic crack growth history for mixed-mode loading case $\left(-150 \leq \theta^{\prime} \leq 150\right)$ to extract stress intensity factors. Figure 21 shows thus extracted stress intensity factor histories.

In Fig. 21, initially both mode-I and -II stress intensity factors are negative. Though unintuitive, the former is caused by a tendency for notch (initially $\sim 300 \mu \mathrm{m}$ wide) to close due to eccentric loading above the crack line as shown in Fig. 11. With the passage of time, upon reflection of compressive stress waves as tensile waves from the free edges (particularly from the cracked edge) of the sample, the crack flanks open (and slide simultaneously) causing mode-I stress intensity factor to turn positive. Mode-II

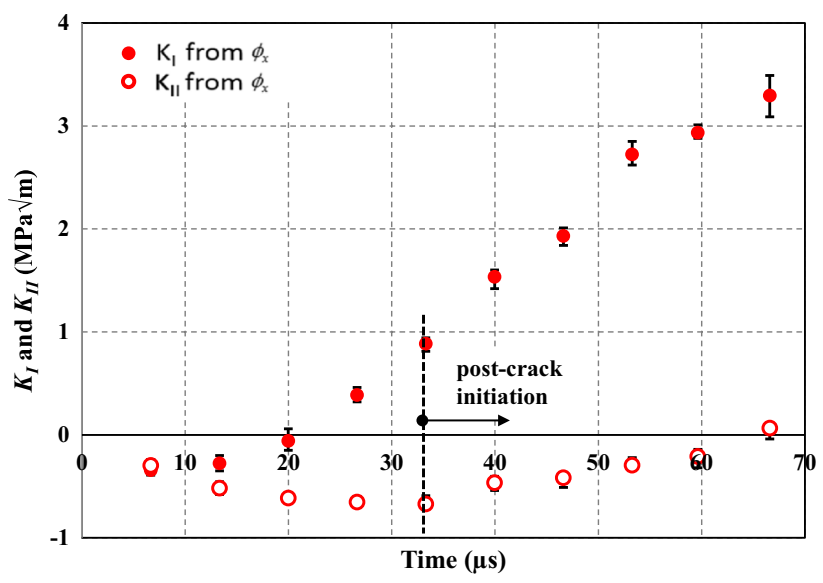

Fig. 21 Dynamic mixed-mode stress intensity factor histories from regression analysis of surface slope data (symbols) 
stress intensity factor continue to be negative, consistent with the loading configuration. Further, its magnitude increases monotonically until crack initiation. After initiation, however, the magnitude of mode-II stress intensity factors decreases to zero as the crack kinks and re-orients to propagate in an increasingly dominant mode-I condition.

\section{Conclusions}

In this work, the reflection-mode digital gradient sensing (r-DGS) method has been successfully extended to the study of static and dynamic fracture mechanics problems. First, it is demonstrated for quasi-static mode-I and mixedmode (I/II) problems using edge cracked PMMA samples. Three-point bend configurations with straight and inclined edge cracks in otherwise symmetrically loaded samples. Two orthogonal surface slopes in the crack-tip vicinity with and without coordinate transformation are used for extracting stress intensity factors. The measurements based on over deterministic least-squares analysis of optical data in conjunction with crack-tip field descriptions have produced reliable fracture parameter estimates.

Next, the r-DGS method has been extended to study dynamic fracture problems under impact loading conditions. The orthogonal deformations near the tip of dynamically loaded stationary cracks as well as transiently propagating cracks are mapped by coupling r-DGS method with highspeed digital photography. The results presented include both self-similar and kinked crack growth relative to the initial crack orientation. The evolution of both mode-I and mixedmode (I/II) stress intensity factor histories have been analyzed using measured optical data in conjunction with plane stress elasto-dynamic steady-state crack-tip field descriptions. The mode-I stress intensity factor history thus obtained matches the one evaluated from a companion elasto-dynamic finite element analysis of the sample based on independently measured transient particle velocity input. The stress intensity factor histories in the mixed-mode case conforms well with the physical aspects of loading the specimen both before and after crack initiation including a precipitous drop in the magnitude of mode-II stress intensity factor after crack initiation.

Acknowledgments The partial support for this research through grants from the National Science Foundation (Grant \#1232821) and Department of Defense (Grant \# W31P4Q-14-C-0049) are gratefully acknowledged.

\section{References}

1. Wells AA, Post D (1958) The dynamic stress distribution surrounding a running crack-a photoelastic analysis. Proc SESA 16(1):69-92
2. Irwin GR (1958) Discussion of the dynamic stress distribution surrounding a running crack - a photoelastic analysis. Proc SESA 16(1):93-96

3. Dally JW (1979) Dynamic photoelastic studies of fracture. Exp Mech 19(10):349-361

4. Ramulu M, Kobayashi AS (1983) Dynamic crack curving-a photoelastic investigation. Exp Mech 23(1):1-9

5. Singh RP, Shukla A (1996) Subsonic and intersonic crack growth along a bimaterial interface. J Appl Mech 63:919-924

6. Post D, Han B, Ifju P (1994) High Sensitivity Moiré. SpringerVerlag, New York

7. Chiang FP, Hareesh TV (1988) Three dimensional crack-tip deformations: an experimental study and comparison to HRR field. Int J Fract 36:243-257

8. Kokaly MT, Lee J, Kobayashi AS (2003) Moire interferometry for dynamic fracture study. Opt Lasers Eng 40:231-247

9. Beinert J, Kalthoff JF (1981) Experimental Determination of Dynamic Stress Intensity Factors by Shadow Patterns. In: Sih GC (ed) Mechanics of fracture, vol 7., Experimental Fracture MechanicsMartinus Nijhoff Publishers, The Hague, Boston, London, pp 280-330

10. Rosakis AJ, Ravi-Chandar K (1986) On crack-tip stress state: an experimental evaluation of three-dimensional effects. Int J Solids Struct 22:121-134

11. Huntley JM, Field JE (1988) Measurement of crack-tip displacement field using laser speckle photography. Eng Fract Mech 30(6):779-790

12. Dudderar TD (1969) Applications of holography to fracture mechanics. Exp Mech 9(6):281-285

13. Washabagh PG, Knauss WG (1994) A reconciliation of dynamic crack growth velocity and Rayleigh wave speed in isotropic brittle solids. Int J Fract 65:97-114

14. Toh SL, Shang HM, Chau FS, Tay CJ, Tay TE (1991) Damage detection using holography and shearography. Fracture of Engineering Materials and Structures. Springer, Netherlands, pp 489-496

15. Tippur HV, Krishnaswamy S, Rosakis AJ (1991) Optical mapping of crack tip deformations using the methods of transmission and reflection coherent gradient sensing: a study of crack tip K-dominance. A coherent gradient sensor for crack-tip deformation measurements: analysis and experimental results. Int $\mathrm{J}$ Fract 52:91-117

16. Rousseau C-E, Tippur HV (2001) Dynamic fracture of compositionally graded materials with cracks along the elastic gradient: experiments and analysis. Mech Mater 33(7):403-421

17. Tippur HV (2010) Coherent gradient sensing (CGS) method for fracture mechanics: a review. Fatigue Fract Eng Mater 33(12):832-858

18. Chu TC, Ranson WF, Sutton MA (1985) Applications of digital image correlation techniques to experimental mechanics. Exp mech 25(3):232-244

19. Sutton MA, Orteu U, Schreier H (2009) Image correlation for shape, motion and deformation measurements. Springer, New York

20. Chiang FP, Asundi A (1979) White light speckle method of experimental strain analysis. Appl Opt 18(4):409-411

21. Chen DJ, Chiang FP, Tan YS, Don HS (1993) Digital speckledisplacement measurement using a complex spectrum method. Appl Opt 32(11):1839-1849

22. Periasamy C, Tippur HV (2012) A full-field digital gradient sensing method for evaluating stress gradients in transparent solids. Appl Opt 51(12):2088-2097

23. Periasamy C, Tippur HV (2013) Measurement of orthogonal stress gradients due to impact load on a transparent sheet using digital gradient sensing method. Exp Mech 53(1):97-111

24. Periasamy C, Tippur HV (2013) A full-field reflection-mode digital gradient sensing method for measuring orthogonal slopes and curvatures of thin structures. Meas Sci Technol 24:\#025202 
25. Jain AS, Tippur HV (2015) Reflection-mode digital gradient sensing method for visualizing and quantifying transient deformations and damage in solids, submitted

26. Jain AS (2015) Extension of a reflection-mode digital gradient sensor to study impact induced deformations, damage, and fracture. M.S. Thesis, Auburn University
27. Kirugulige MS, Tippur HV (2006) Mixed-mode dynamic crack growth in functionally graded glass-filled epoxy. Exp Mech 46(2):269-281

28. Anderson TL (1995) Fracture mechanics, fundamentals and applications, 2nd edn. CRC Press, Boca Raton 